\title{
Two-Equation Eddy-Viscosity Turbulence Models for Engineering Applications
}

\author{
F. R. Menter* \\ NASA Ames Research Center, Moffett Field, California 94035
}

\begin{abstract}
Two new two-equation eddy-viscosity turbulence models will be presented. They combine different elements of existing models that are considered superior to their alternatives. The first model, referred to as the baseline (BSL) model, utilizes the original $k-w$ model of Wilcox in the inner region of the boundary layer and switches to the standard $k-\epsilon$ model in the outer region and in free shear flows. It has a performance similar to the Wilcox model, but avoids that model's strong freestream sensitivity. The second model results from a modification to the definition of the eddy-viscosity in the BSL model, which accounts for the effect of the transport of the principal turbulent shear stress. The new model is called the shear-stress transport-model and leads to major improvements in the prediction of adverse pressure gradient flows.
\end{abstract}

\section{Introduction}

$\mathbf{T}$ HIS paper is concerned with two-equation eddy-viscosity turbulence models with emphasis on an engineering perspective. It is based on the experience of the author in testing a large number of turbulence models against a wide variety of experimental test cases. The test flows cover a significant range of flow situations typically encountered in aerodynamic computations and are believed to allow some conclusions about a model's ability to perform in engineering applications. Two new turbulence models will be presented. They are based on a combination of what the author believes to be the best elements of existing eddy-viscosity models.

There is a discrepancy between the large number of publications about two-equation models and the slow pace of improvement in accuracy that has been achieved since their introduction. The basic problem of two-equation models, namely, their failure to correctly predict the onset and amount of separation in adverse pressure gradient flows, is still unresolved. Furthermore, there is no agreement on the standards by which to measure the improvement achieved by proposed new models, or alterations to existing models. Many times new models are based on theoretical concepts, which by themselves involve severe assumptions about the nature of turbulence, not even approximately satisfied in aerodynamic flows (homogeneous turbulence, small pressure gradients, low Reynolds number, flow equilibrium, etc.). It has been the author's experience that small changes $(5-10 \%)$ in modeling constants can lead to a significant improvement (or deterioration) of model predictions. None of the available theoretical tools (dimensional analysis, asymptotic expansion theory, use of direct numerical simulations (DNS) data, renormalization group (RNG) theory, rapid distortion theory, etc.) can provide constants to that degree of accuracy. The only way to establish the validity of theoretical arguments under those conditions is to carefully test the resulting model against a number of challenging and well-documented research flows. Unfortunately, this is not general practice, and it is often unclear whether the improvements presented for one type of flow

Presented as Paper 93-2906 at the AIAA 23rd Fluid Dynamics, Plasmadynamics, and Lasers Conference, Orlando, FL, July 6-9, 1993; received July 26, 1993; revision received Dec. 30, 1993; accepted for publication Jan. 26, 1994. Copyright (c) 1993 by the American Institute of Aeronautics and Astronautics, Inc. No copyright is asserted in the United States under Title 17, U.S. Code. The U.S. Government has a royalty-free license to exercise all rights under the copyright claimed herein for Governmental purposes. All other rights are reserved by the copyright owner.

*Research Scientist, Fluid Dynamics Division, MS 229-1. (e.g., boundary-layer flows) will not lead to a deterioration for another class of equally important flows (e.g., free shear flows). The author feels that the slow progress in engineering turbulence modeling, and the confusing picture it often presents, result to no small extent from an overemphasis of theoretical concepts and a virtual denial of the empirical nature of the subject.

Following an empirical approach, the author has developed two new turbulence models based on elements of existing models which are considered to be superior to their alternatives. A description of these new models follows as well as an explanation of the rationale behind the choices that have been made in different areas of the flow and an address to anticipated criticism.

The $k-\omega$ model $^{1}$ is the model of choice in the sublayer of the boundary layer. Unlike any other two-equation model, the $k-\omega$ model does not involve damping functions and, as will be shown, allows simple Dirichlet boundary conditions to be specified. Because of its simplicity, the $k-\omega$ model is superior to other models, especially with regard to numerical stability. Furthermore, it is as accurate as any other model in predicting the mean flow profiles. Wilcox ${ }^{1}$ has developed modifications that allow the treatment of rough walls and surface mass injection which can be used in the new model without change.

One point of criticism is that the $k-\omega$ model (like many other models) does not correctly predict the asymptotic behavior of the turbulence as it approaches the wall. However, the Taylor series expansion of the Navier-Stokes equations that underlies the analysis is only valid in the immediate wall proximity. So close to the surface the eddy viscosity is much smaller than the molecular viscosity and the asymptotic behavior of the mean flow profile is independent of the asymptotic form of the turbulence. Therefore, even if the turbulence model is not asymptotically consistent, the mean flow profile and the wall skin friction are still predicted correctly. A second point of criticism is that the $k-\omega$ model does not accurately represent the $k$ and $\epsilon$ distribution in agreement with DNS data. A significant number of damping functions have been developed in the last years for the $k-\epsilon$ model which lead to an improved agreement with DNS data. In Refs. 2 and 3, a number of $k-\epsilon$ models with different damping functions have been tested for a significant number of flows, with the conclusion that the specific form of the damping functions has little to no effect on the predicted velocity profiles and the skin friction of high-Reynolds-number flows. It should not be forgotten that the main (and often the only) information the mean flow solver gets from the turbulence model is the eddy viscosity. It is not clear why fitting the DNS data for $k$ and $\epsilon$ should lead to an improved eddy-viscosity distribution. In the end, the 
agreement with DNS data might only be a matter of interpretation. In the sublayer, Wilcox equates the quantity $k$ in his model as being proportional to the normal component (with respect to the wall) of the turbulent kinetic energy. This interpretation leads to a very good agreement with experimental and DNS data. In cases where the agreement with DNS data is considered important, the damping functions developed by Wilcox ${ }^{4}$ can be applied to the present model.

The $k-\omega$ model is also used in the logarithmic part of the boundary layer. It has been shown $n^{1,5}$ that the behavior of the $k$ - $\omega$ model in the logarithmic region is superior to that of the $k-\epsilon$ model in equilibrium adverse pressure gradient flows and in compressible flows.

In the wake region of the boundary layer, the $k-\omega$ model has to be abandoned in favor of the $k-\epsilon$ model. The reason for this switch is that the $k-\omega$ model has a very strong sensitivity to the (quite arbitrary) freestream values $\omega_{f}$ specified for $\omega$ outside the boundary layer. It has been shown in Ref. 6 that the eddy viscosity in boundary and free shear layers can be changed by more than $100 \%$ by simply reducing the value of $\omega_{f}$. It has also been shown in Ref. 6 that the $k-\epsilon$ model does not suffer from this deficiency. There is no mathematical theory to date which distinguishes between two-equation models that suffer from the freestream dependency and those that do not. It is therefore of great importance that the influence of freestream values on the solutions of newly developed models is tested very carefully.

The mathematical analysis of the behavior of two-equation models in adverse pressure gradient flows has been largely restricted to the logarithmic region. ${ }^{1,7}$ Although the behavior of the model in the logarithmic region is of importance, especially in flows with moderate pressure gradients, it is the level of the eddy viscosity in the wake region that ultimately determines the ability of an eddy-viscosity model to predict strong adverse pressure gradient flows. This has been clearly demonstrated by the improvement that the Johnson-King model ${ }^{8}$ achieved over standard algebraic models by reducing the wake region eddy viscosity in adverse pressure gradient flows. The limited influence of the logarithmic region on the results for strong adverse pressure gradients is also evident in the failure of the original $k-\omega$ model to accurately predict pressure-induced separation (as will be shown later) despite its superior log-region characteristics. The basic idea behind the JohnsonKing model is to enforce Bradshaw's observation that the principal turbulent shear stress is proportional to the turbulent kinetic energy in the wake region of the boundary layer. Enforcing this proportionality introduces a lag effect into the equations that accounts for the transport of the principal turbulent shear stress. It will be shown that the classical formulation of the eddy viscosity in two-equation models violates Bradshaw's relation and thereby misses this important effect. In the new model the eddy-viscosity formulation will be modified to take the transport effects into account.

Finally, in free shear layers away from surfaces, the standard $k-\epsilon$ model will be utilized. There does not seem to be a model that accurately predicts all free shear flows (wake, jet, mixing layer) and the $k-\epsilon$ seems to be a fair compromise.

To achieve the desired features in the different regions, the standard high-Reynolds-number version of the $k-\epsilon$ model will be transformed to a $k$ - $\omega$ formulation. It will then be multiplied by a blending function $\left(1-F_{1}\right)$ and added to the original $k-\omega$ model times $F_{1}$. The blending function $F_{1}$ will be designed to be one in the sublayer and logarithmic region of the boundary layer and to gradually switch to zero in the wake region. This means that the new model will be based on a $k-\omega$ formulation, with the original Wilcox model activated in the near wall region and the standard $k-\epsilon$ model activated in the outer wake region and in free shear layers. This first step leads to a new model that will be termed the baseline (BSL) model. The BSL model has a performance very similar to that of the original $k-\omega$ model, but without the undesirable freestream dependency.

In a second step, the definition of the eddy viscosity will be modified to account for the transport of the principal turbu- lent shear stress. The resulting model will be called the shearstress transport (SST) model. It is this second step that leads to a major improvement in performance over both the original $k-\omega$ and the standard $k-\epsilon$ model.

\section{Turbulence Model}

\section{New Baseline Model}

The idea behind the BSL model is to retain the robust and accurate formulation of the Wilcox $k-\omega$ model in the near wall region, and to take advantage of the freestream independence of the $k-\epsilon$ model in the outer part of the boundary layer. To achieve this, the $k-\epsilon$ model is transformed into a $k-\omega$ formulation. The difference between this formulation and the original $k-\omega$ model is that an additional cross-diffusion term appears in the $\omega$ equation and that the modeling constants are different (A small additional diffusion term is neglected in the transformation. It is shown in Ref. 9 that the term has virtually no effect on the solutions). The original model is then multiplied by a function $F_{1}$ and the transformed model by a function $\left(1-F_{1}\right)$, and both are added together. The function $F_{1}$ will be designed to be one in the near wall region (activating the original model) and zero away from the surface. The blending will take place in the wake region of the boundary layer. The left-hand side of the following equations is the Lagrangian derivative: $\mathrm{D} / \mathrm{D} t:=\partial / \partial t+u_{i} \partial / \partial x_{i}$.

Original $k-\omega$ model:

$$
\begin{aligned}
& \frac{\mathrm{D} \rho k}{\mathrm{D} t}=\tau_{i j} \frac{\partial u_{i}}{\partial x_{j}}-\beta^{*} \rho \omega k+\frac{\partial}{\partial x_{j}}\left[\left(\mu+\sigma_{k 1} \mu_{t}\right) \frac{\partial k}{\partial x_{j}}\right] \\
& \frac{\mathrm{D} \rho \omega}{\mathrm{D} t}=\frac{\gamma_{1}}{\nu_{t}} \tau_{i j} \frac{\partial u_{i}}{\partial x_{j}}-\beta_{1} \rho \omega^{2}+\frac{\partial}{\partial x_{j}}\left[\left(\mu+\sigma_{\omega 1} \mu_{t}\right) \frac{\partial \omega}{\partial x_{j}}\right]
\end{aligned}
$$

Transformed $k-\epsilon$ model:

$$
\begin{aligned}
& \frac{\mathrm{D} \rho k}{\mathrm{D} t}=\tau_{i j} \frac{\partial u_{i}}{\partial x_{j}}-\beta^{*} \rho \omega k+\frac{\partial}{\partial x_{j}}\left[\left(\mu+\sigma_{k 2} \mu_{t}\right) \frac{\partial k}{\partial x_{j}}\right] \\
& \frac{\mathrm{D} \rho \omega}{\mathrm{D} t}=\frac{\gamma_{2}}{\nu_{t}} \tau_{i j} \frac{\partial u_{i}}{\partial x_{j}}-\beta_{2} \rho \omega^{2}+\frac{\partial}{\partial x_{j}}\left[\left(\mu+\sigma_{\omega 2} \mu_{t}\right) \frac{\partial \omega}{\partial x_{j}}\right] \\
& +2 \rho \sigma_{\omega 2} \frac{1}{\omega} \frac{\partial k}{\partial x_{j}} \frac{\partial \omega}{\partial x_{j}}
\end{aligned}
$$

Now, Eq. (1) and Eq. (2) are multiplied by $F_{1}$ and Eq. (3) and Eq. (4) are multiplied by $\left(1-F_{1}\right)$ and the corresponding equations of each set are added together to give the new model:

$$
\begin{gathered}
\frac{\mathrm{D} \rho k}{\mathrm{D} t}=\tau_{i j} \frac{\partial u_{i}}{\partial x_{j}}-\beta^{*} \rho \omega k+\frac{\partial}{\partial x_{j}}\left[\left(\mu+\sigma_{k} \mu_{t}\right) \frac{\partial k}{\partial x_{j}}\right] \\
\frac{\mathrm{D} \rho \omega}{\mathrm{D} t}=\frac{\gamma}{\nu_{t}} \tau_{i j} \frac{\partial u_{i}}{\partial x_{j}}-\beta \rho \omega^{2}+\frac{\partial}{\partial x_{j}}\left[\left(\mu+\sigma_{\omega} \mu_{t}\right) \frac{\partial \omega}{\partial x_{j}}\right] \\
+2 \rho\left(1-F_{1}\right) \sigma_{\omega 2} \frac{1}{\omega} \frac{\partial k}{\partial x_{j}} \frac{\partial \omega}{\partial x_{j}}
\end{gathered}
$$

Let $\phi_{1}$ represent any constant in the original model $\left(\sigma_{k 1}, \ldots\right)$, $\phi_{2}$ any constant in the transformed $k-\epsilon$ model $\left(\sigma_{k 2}, \ldots\right)$ and $\phi$ the corresponding constant of the new model $\left(\sigma_{k} \ldots\right)$, then the relation between them is:

$$
\phi=F_{1} \phi_{1}+\left(1-F_{1}\right) \phi_{2}
$$

All constants, as well as the function $F_{1}$, are given in the Appendix.

\section{Shear-Stress Transport Model}

One of the major differences between eddy-viscosity and full Reynolds-stress models, with respect to aerodynamic applications, is that the latter accounts for the important effect of the 
transport of the principal turbulent shear stress $\tau=:-\rho \overline{u^{\prime} v^{\prime}}$ (obvious notation) by the inclusion of the term

$$
\frac{\mathrm{D} \tau}{\mathrm{D} t}=: \frac{\partial \tau}{\partial t}+u_{k} \frac{\partial \tau}{\partial x_{k}}
$$

The importance of this term has clearly been demonstrated by the success of the Johnson-King (JK) model. ${ }^{8}$ Note that the main difference between the JK model and the Cebeci-Smith model lies in the inclusion of this term in the former, leading to significantly improved results for adverse pressure gradient flows. The JK model features a transport equation for the turbulent shear stress $\tau$ that is based on Bradshaw's assumption that the shear stress in a boundary layer is proportional to the turbulent kinetic energy $k$ :

$$
\tau=\rho a_{1} k
$$

with $a_{1}$ being a constant. On the other hand, in two-equation models, the shear stress is computed from:

$$
\tau=\mu_{t} \Omega
$$

with $\Omega=(\partial u / \partial y)$. For conventional two-equation models, Eq. (10) can be rewritten to give:

$$
\tau=\rho \sqrt{\frac{\text { Production }_{k}}{\text { Dissipation }_{k}}} a_{1} k
$$

as noted in Ref. 10. In adverse pressure gradient flows the ratio of production to dissipation can be significantly larger than one, as found from the experimental data of Driver, ${ }^{11}$ and therefore Eq. (11) leads to an overprediction of $\tau$. To satisfy Eq. (9) within the framework of an eddy-viscosity model, the eddy viscosity is redefined in the following way:

$$
\nu_{t}=\frac{a_{1} k}{\max \left(a_{1} \omega ; \Omega F_{2}\right)}
$$

where $F_{2}$ is a function that is one for boundary-layer flows and zero for free shear layers. In an adverse pressure gradient boundary layer, production of $k$ is larger than its dissipation (or $\Omega>a_{1} \omega$ ) and Eq. (12) therefore guarantees that Eq. (9) is satisfied whereas the original formulation $\nu_{t}=k / \omega$ is used for the rest of the flow.

To recover the original formulation of the eddy viscosity for free shear layers [where Bradshaw's assumption, expressed in Eq. (9) does not necessarily hold] the modification to the shear-stress transport (SST) model is limited to wall bounded flows. This is achieved in the same way as it is for the BSL model by applying a blending function $F_{2}$ (also defined in the appendix). For general flows $\Omega$ is taken to be the absolute value of the vorticity.

\section{Model Versatility and Generality}

The price for avoiding the freestream dependency and achieving the improved performance due to the modification in the eddy viscosity lies mainly in the necessary computation of the blending functions $F_{1}, F_{2}$ and the additional cross-diffusion term. The blending functions involve the distance from the surface which, however, has to be computed only once (as long as there is no grid deformation). Note that the distance from the surface is uniquely defined as being the shortest distance between the present point and all no-slip boundaries (distance does not have to be measured normal to a surfacee.g., backward-facing step). In most application codes, the boundary points have a marker and the computation of the distance function can therefore be automated. The increase in complexity from the Wilcox model to the present model is mainly in terms of coding. The overall computing time, as well as the stability of the code are not affected.

Once the model is implemented, it offers a wide variety of options. An example is a two-layer $k-\epsilon$ model $^{12}$ with the original $k-\omega$ model in the sublayer and the $k-\epsilon$ model in the highReynolds-number region. This can be achieved by changing the argument of $F_{1}$ for the BSL model from Eq. (A9) (see Appendix) to:

$$
\arg _{1}=\min \left(\frac{500 \nu}{y^{2} \omega} ; \frac{4 \rho \sigma_{\omega 2} k}{C D_{k \omega} y^{2}}\right)
$$

The modification ensures that $F_{1}$ is zero for $y^{+}>70$. This two-layer $k-\epsilon$ model utilizes the superior sublayer characteristics of the $k-\omega$ model in much the same way that the model in Ref. 12 introduces an algebraic expression into the $\epsilon$ equation. However, in the present approach the blending between the two regions is performed automatically and without user input.

The versatility of the model makes it possible to give the user a number of options, without making it necessary to program various models.

\section{Numerical Method}

The mean flow equations are solved by the INS3D code of Rogers and $\mathrm{Kwak}^{13}$ which is based on a pseudocompressibility method. Important details about the discretization of the turbulence model are given in Ref. 9. All computations have been performed on different grids to ensure that the presented solutions are grid independent. The airfoil computations were performed on a standard grid kindly provided by Rogers. ${ }^{14}$ The standard $k-\epsilon$ model is coded as given in Ref. 15 .

\section{Results}

\section{Flat Plate Boundary Layer}

To demonstrate the freestream dependency of the original $k-\omega$ model, flat plate zero pressure gradient boundary-layer computations with different freestream values for $\omega$ have

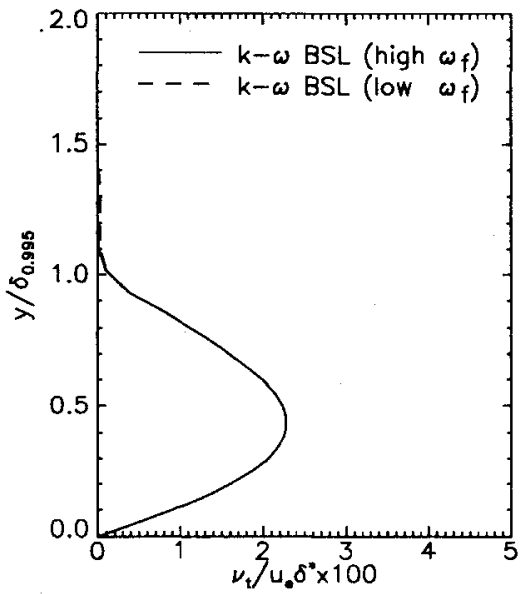

Fig. 1 Freestream dependency of the eddy viscosity for the original and the BSL $k-\omega$ model. 


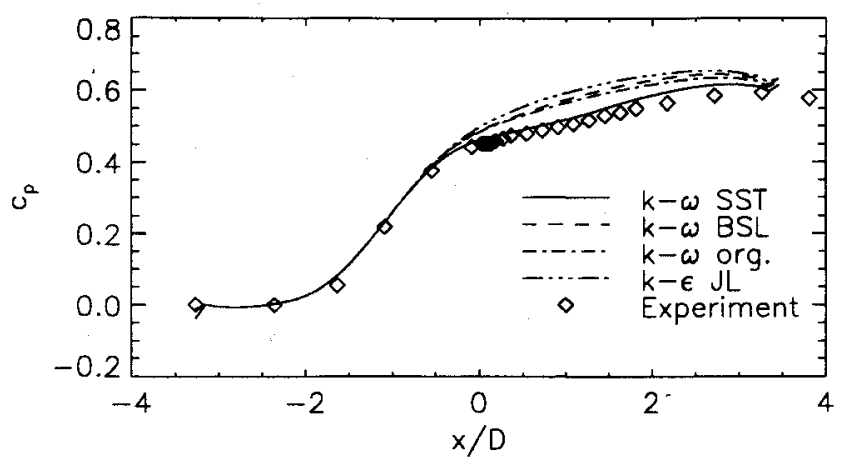

Fig. 2 Wall pressure distribution for Driver's adverse pressure-gradient flow.

been performed. For the first set of computations, the correct freestream values as given in Ref. 6 have been specified at the inflow boundary freestream for both the original and the BSL $k-\omega$ model. Then, the preceding value was reduced by four orders of magnitude and the computations were repeated with both models. Note that the freestream value of $k$ was also reduced to keep the freestream value of the eddy-viscosity constant (the freestream value of the eddy viscosity has no influence, as long as it is small compared to its values inside the boundary layer). Figure 1 shows eddy-viscosity profiles for the original and the BSL $k-\omega$ model. The eddy viscosity of the original model changes by almost $100 \%$ due to the changes in $\omega_{f}$, whereas the BSL model gives the same results for both cases. The strong sensitivity of the original model to $\omega_{f}$ is clearly unacceptable and can lead to a severe deterioration of the results for complex flows, as will be shown later. Results of the SST model are also independent of $\omega_{f}$. A more detailed study of the freestream dependency can be found in Refs. 6 and 9.

In each of the following comparisons between the different models, $\omega_{f}$ was always chosen according to the formula given in Ref. 6.

Zero pressure gradient flat plate boundary layer computations are given in Ref. 9. All models give good agreement with the experimental correlations for $u^{+}$versus $y^{+}$and $c_{f}$. The $k-\omega$ models can be run with the first gridpoint as far out as $y^{+}=3$ without a deterioration of the results.

\section{Free Shear Layers}

For free shear layers the SST and the BSL models reduce to the same model $\left(F_{1}=0 ; F_{2}=0\right)$, and are virtually identical to the standard $k-\epsilon$ model. Because the behavior of the $k-\epsilon$ model for free shear layers is well known, and because of space limitations, results are not shown here, but can be found in Ref. 9. Reference 9 also shows the ambiguity of the results of the original $k-\omega$ model $^{1}$ with respect to the freestream values.

\section{Adverse Pressure Gradient Flows}

One of the most important aspects of a turbulence model for aerodynamic applications is its ability to accurately predict adverse pressure gradient boundary-layer flows. It is especially important that a model be able to predict the location of flow separation and the displacement effect associated with it.

The test case most widely used to measure the performance of turbulence models under adverse pressure gradient conditions is the flow reported by Samuel and Joubert. ${ }^{16}$ Results for this flow are shown in Ref. 9 and are not reproduced here due to space limitations. It was found in Ref. 9 that all three $k-\omega$ models reproduce the experimental data very well, whereas the JL $k-\epsilon$ model gives values that are too high for $c_{f}$.

The small differences between the solutions reported in Ref. 9 , especially between the different $k-\omega$ models, do not allow final conclusions about the abilities of the models to predict adverse pressure gradient flows. It appears that the SamuelJoubert flow does not pose a sufficiently strong challenge to the models to assess their potentials for these types of flows. The author has reached a similar conclusion in Ref. 10 . It is therefore important to test models under more demanding conditions, with stronger adverse pressure gradients and possibly separation. The following flowfield, reported by Driver, ${ }^{11}$ has proven to be a highly self-consistent and demanding test case.

In Driver's flow, a turbulent boundary layer develops in the axial direction of a circular cylinder. An adverse pressure gradient is imposed by diverging wind tunnel walls and suction applied at these walls. The pressure gradient is strong enough to cause the flowfield to separate. The inflow Reynolds number is $2.8 \cdot 10^{5}$ based on the diameter $D$ of the cylinder (140 $\mathrm{mm})$. A $60 \times 3 \times 60$ grid $^{10}$ was used for the present computations. A computation on a $100 \times 3 \times 100$ grid gave almost identical results.

Figure 2 shows the wall pressure distribution for Driver's flow as computed by the different models. The SST model gives superior results to the other models due to its ability to account for the transport of the principal turbulent shear stress. As expected, the JL $k-\epsilon$ model produces the least accurate results, with the BSL and the original $k-\omega$ model being close to each other in the middle.

Figure 3, depicting the wall shear-stress distribution for Driver's flow, shows that the SST model predicts the largest amount of separation, whereas the JL model stays firmly attached. Again, the BSL and the original $k-\omega$ model produce very similar results.

The differences between the models can be seen in Fig. 4, which shows the velocity profiles. The SST model clearly produces the best agreement with the experiments. The larger displacement effect predicted by this model is reflected in the flattening of the $c_{p}$ distribution as was observed in Fig. 2. The original $k-\omega$ model predicts slightly better results than the BSL model, and the JL $k-\epsilon$ model shows very little sensitivity to the pressure gradient, as was already reflected in Figs. 2 and 3.

The reasons for the different behavior of the models can be seen in the following two pictures. Figure 5 compares turbulent shear-stress profiles at different stations. The JL model

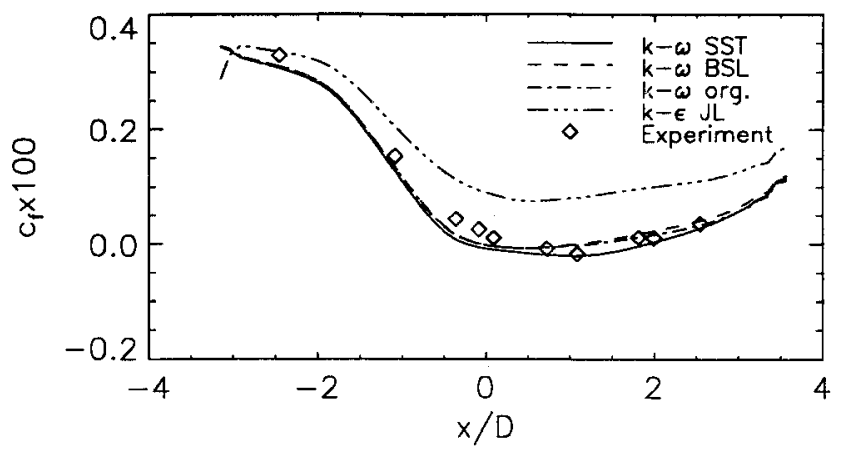

Fig. 3 Wall shear-stress distribution for Driver's adverse pressuregradient flow.

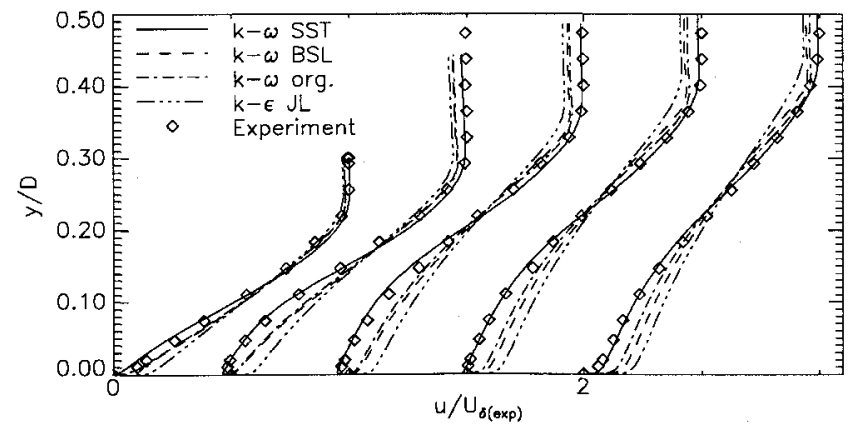

Fig. 4 Velocity profiles for Driver's adverse pressure-gradient flow at $x / D=-0.091,0.363,1.088,1.633$, and 2.177. 
obviously predicts significantly higher shear-stress levels than the other models in the region where separation is approached. This in turn leads to the firmly attached velocity profiles of Fig. 4. The differences between the models can also be seen from the eddy-viscosity distributions. Figure 6 shows the maximum value of the kinematic eddy-viscosity profiles for all streamwise $(x)$ stations, nondimensionalized by $u_{e} \delta^{*}$. The SST model predicts the reduction of this quantity due to the adverse pressure gradient in very good agreement with the experiments. The BSL and the original $k-\omega$ model are very close to each other up to separation (around $x / D=0$ ), whereas the original model is closer to the experiments in the recovery region. Both models give consistently too high values for the maximum eddy viscosity in the adverse pressure gradient region. The $k-\epsilon$ model falls only barely below the value of 0.0168 recommended by Clauser for equilibrium boundary layers (and used in the Cebeci-Smith model) and thereby fails to account for the nonequilibrium effects altogether.

\section{Backward-Facing Step Flow}

Results for the flow over a backward-facing step as reported by Driver and Seegmiller ${ }^{17}$ will be discussed next. This flowfield was a test case in the 1981 Stanford conference for the evaluation of turbulence models. However, most of the computations at the time were performed on comparatively coarse grids and there is substantial evidence that significantly finer grids have to be used to obtain grid-independent results. ${ }^{18}$ The present computations have been performed on a $120 \times 120$ grid, with substantial grid refinement near the step. As with the other flowfields, a grid refinement study was made. The present results are virtually identical to those performed on a $90 \times 90$ and on a $240 \times 240$ grid.

Figure 7 shows a comparison of computed and experimental skin friction distributions. The $k-\omega$ models all perform significantly better than the $k-\epsilon$ model. The reattachment length of

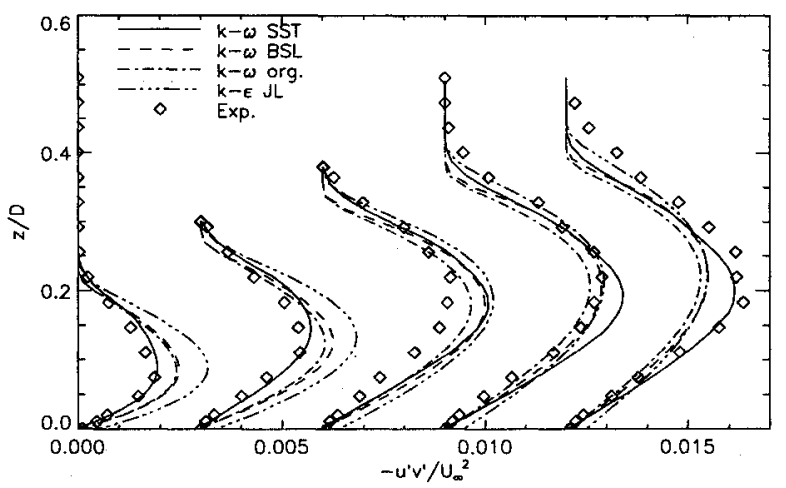

Fig. 5 Turbulent shear-stress profiles for Driver's adverse pressuregradient flow at $x / D=-0.091,0.363,1.088,1.633$, and 2.177.

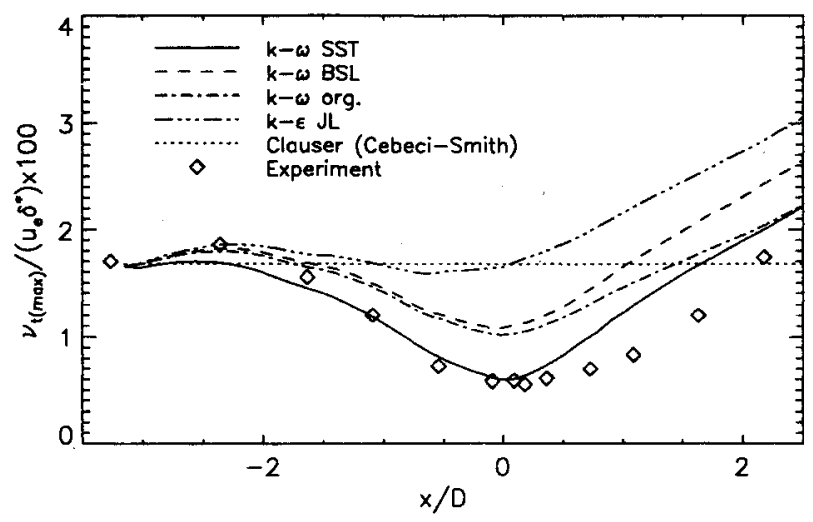

Fig. $6 \nu_{t}(\max ) / u_{e} \delta *$ distribution for Driver's adverse pressure-gradient flow.

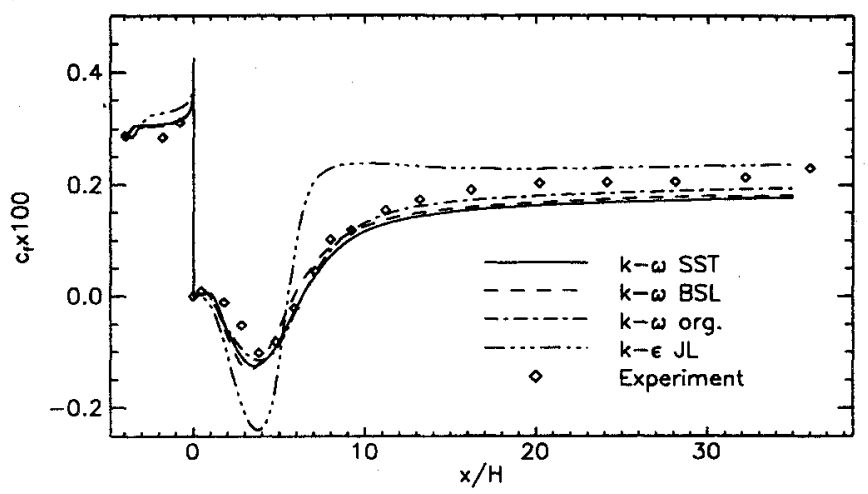

Fig. 7 Wall shear-stress distribution for backward-facing step flow.

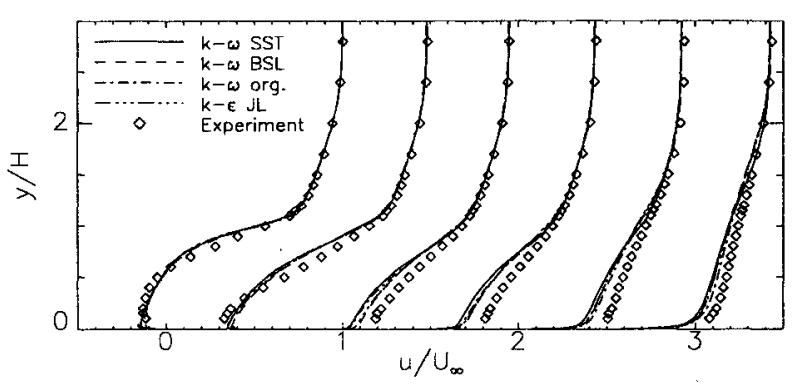

Fig. 8 Velocity profiles for backward-facing step flow at the streamwise locations: $x / H=2.0,4.0,6.5,8.0,14.0$, and 32.0 .

the four models are 6.5 (SST), 5.9 (BSL), 6.4 (original $k-\omega$ ), and 5.5 (JL $k-\epsilon)$ compared to a value of about 6.4 in the experiments. The reattachment length predicted by the $k-\epsilon$ model is better than previously reported, certainly as a result of the fine grid employed in the present computations (see also Ref. 18). However, the model predicts variations of $c_{f}$ which are significantly too large in the recirculation and the reattachment region.

Figure 8 shows a comparison of the velocity profiles. All models fail to capture the relaxation downstream of reattachment correctly. The results of Ref. 19 show that this is also true for a more complex model which accounts for anisotropy effects.

\section{NACA 4412 Airfoil Flow}

The following set of computations is for the flow around a NACA 4412 airfoil at 13.87 deg angle of attack. The Reynolds number with respect to the chord length is $R e=1.52 \cdot 10^{6}$. Experimental data for this flow have been reported by Coles and Wadcock..$^{20}$ The grid for the computations consists of $241 \times 61$ points and was made available by Rogers. ${ }^{14}$ It is similar to the one used in Ref. 21.

Figure 9 shows a comparison of the computed and the experimental velocity profiles at different streamwise stations. The results are similar to those for the separated case of Driver, Fig. 4. Again, the SST model predicts the displacement effect in very good agreement with the experiments. The BSL model is showing some response to the pressure gradient, and produces results similar to those reported in Ref. 21 for the Baldwin-Barth model. Another interesting result of this computation is that the original $k-\omega$ model predicts velocity profiles even further away from the experiments than does the Jones-Launder $k-\epsilon$ model. The reason for the poor performance of the original $k-\omega$ model lies in its freestream dependency (for details see Ref. 9). To prove this point, Fig. 9 also shows computations for the SST model and the original $k-\omega$ model with different freestream values for $\omega$. In the curves labeled with high $\omega_{f}$, the value of $\omega$ was prevented from decaying between the inflow boundary and the leading edge of the airfoil, so that the freestream value $\omega_{f}$ was about fifty 


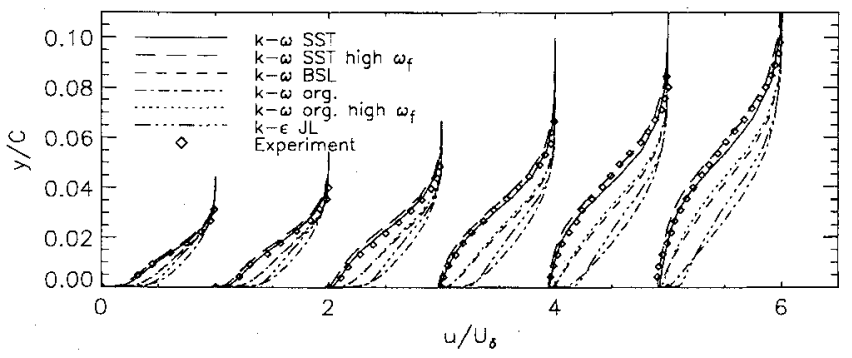

Fig. 9 Velocity profiles on the upper surface of a NACA 4412 airfoil at $13.87 \mathrm{deg}$ angle of attack; streamwise stations $x / c=0.675,0.731$, $0.786,0.842,0.897$, and 0.953 .

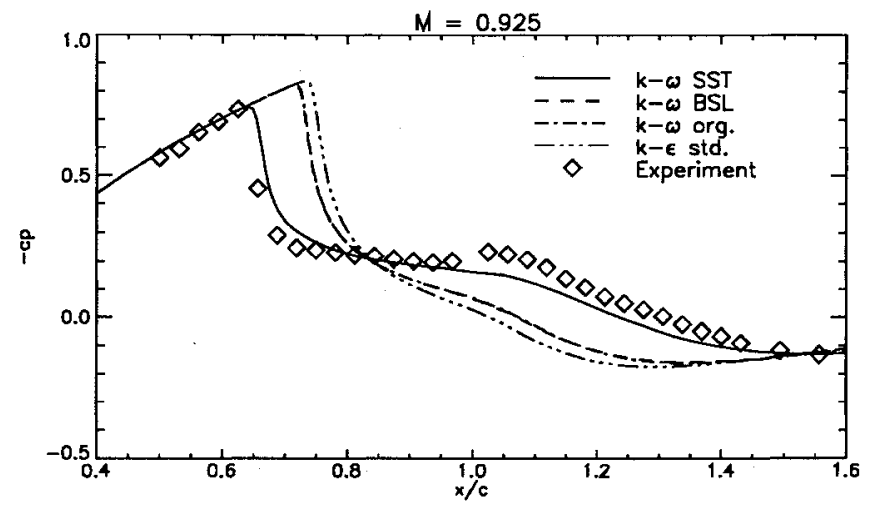

Fig. 10 Comparison of surface pressure distributions for transonic bump flow at $M=0.925$.

times larger than it was in the previous computations. The change in $\omega_{f}$ had very little impact on the computation with the SST model (small changes might be due to a slight influence on the transition behavior), whereas the original $k-\omega$ model predicts significantly different results. The results of the original model for the high $\omega_{f}$ are very close to those of the BSL model, as had to be expected from the derivation of the models. This example clearly shows the dangers of using the original $k-\omega$ model for industrial applications.

\section{Transonic Bump Flow}

The final test case is the axisymmetric transonic shockwave/turbulent boundary-layer experiment of Bachalo and Johnson. ${ }^{22}$ In this experiment, an axisymmetric boundary layer interacts with a shock wave created by a circular arc. It is beyond the scope of this paper to present a detailed study of transonic flows and only the highest Mach number case $(M=0.925)$ will be shown. The number of gridpoints used was $150 \times 3 \times 80$. Grid independence was established by using different grids $(129 \times 3 \times 60$ and $180 \times 3 \times 100)$. Figure 10 shows the wall pressure distribution computed by the different models, compared with the experiment. The SST model predicts significantly better results than the other models, due to its improved transport features. Detailed comparisons for transonic flows will be presented in the future.

\section{Conclusions}

Two new turbulence models have been developed on a strictly empirical basis. They are combinations of what the author considers to be the best available elements of existing eddy-viscosity models to date. Both models are based on a $k-\omega$ formulation which is superior to other formulations with regard to numerical stability. In a first step, a new baseline (BSL) model has been derived. It utilizes the original $k$-w model in the sub- and log-layer and gradually switches to the standard $k-\epsilon$ model in the wake region of the boundary layer. The $k-\epsilon$ model is also used in free shear layers. For boundary- layer flows, the BSL model is very similar to the original $k-\omega$ model, but it avoids the strong freestream sensitivity of that model.

In the second step, a modification to the eddy viscosity has been introduced. It is based on the philosophy underlying the Johnson-King model, which holds that the transport of the principal turbulent shear stress is of vital importance in the prediction of severe adverse pressure gradient flows. The resulting model is termed the shear-stress transport (SST) model.

Both models have been carefully fine tuned and tested for a large number of challenging research flows. The original $k-\omega$, as well as the standard $k-\epsilon$ model are included in the comparison. As expected, the BSL model gives results very close to the original $k-\omega$ model of Wilcox but avoids its freestream dependency. The SST model leads to a significant improvement for all flows involving adverse pressure gradients and should be the model of choice for aerodynamic applications. It is the only available two-equation model that has demonstrated the ability to accurately predict pressure-induced separation and the resulting viscous-inviscid interaction.

The new models require an increased amount of programming effort compared to the original $k$ - $\omega$ model. However, once programmed, the new models consume only insignificantly more computing time and more importantly, they have proven to be very stable even in complex applications. ${ }^{23}$ The concept underlying the new models is very flexible and lends itself to a multitude of different combinations. An example given in the text is a two-layer $k-\epsilon$ model.

It is the author's conviction that a turbulence model has to be tested rigorously for a large number of flows, to establish the boundaries of its usefulness. Because of the limitations of the available theoretical tools and the severe assumptions involved, this is also true for models based on more theoretical arguments. The new models are presently tested for transonic flows with very encouraging results. An early version of the SST model has been tested for complex three-dimensional flows in Ref. 24 . The results compare very favorably with the results of a full Reynolds-stress model, but significantly more testing in three-dimensional flows will be necessary.

\section{Appendix: Baseline and Shear-Stress Transport Models} Baseline Model

$$
\begin{aligned}
& \frac{\mathrm{D} \rho k}{\mathrm{D} t}=\tau_{i j} \frac{\partial u_{i}}{\partial x_{j}}-\beta^{*} \rho \omega k+\frac{\partial}{\partial x_{j}}\left[\left(\mu+\sigma_{k} \mu_{t}\right) \frac{\partial k}{\partial x_{j}}\right] \\
& \frac{\mathrm{D} \rho \omega}{\mathrm{D} t}=\frac{\gamma}{\nu_{t}} \tau_{i j} \frac{\partial u_{i}}{\partial x_{j}}-\beta \rho \omega^{2}+\frac{\partial}{\partial x_{j}}\left[\left(\mu+\sigma_{\omega} \mu_{t}\right) \frac{\partial \omega}{\partial x_{j}}\right] \\
& +2\left(1-F_{1}\right) \rho \sigma_{\omega 2} \frac{1}{\omega} \frac{\partial k}{\partial x_{j}} \frac{\partial \omega}{\partial x_{j}}
\end{aligned}
$$

The constants $\phi$ of the new model are calculated from the constants, $\phi_{1}, \phi_{2}$, as follows:

$$
\phi=F_{1} \phi_{1}+\left(1-F_{1}\right) \phi_{2}
$$

The constants of set $1\left(\phi_{1}\right)$ are (Wilcox):

$$
\begin{gathered}
\sigma_{k 1}=0.5, \quad \sigma_{\omega 1}=0.5, \quad \beta_{1}=0.0750 \\
\beta^{*}=0.09, \quad \kappa=0.41, \quad \gamma_{1}=\beta_{1} / \beta^{*}-\sigma_{\omega 1} \kappa^{2} / \sqrt{\beta^{*}}
\end{gathered}
$$

The constants of set $2\left(\phi_{2}\right)$ are (standard $\left.k-\epsilon\right)$ :

$$
\begin{gathered}
\sigma_{k 2}=1.0, \quad \sigma_{\omega 2}=0.856, \quad \beta_{2}=0.0828 \\
\beta^{*}=0.09, \quad \kappa=0.41, \quad \gamma_{2}=\beta_{2} / \beta^{*}-\sigma_{\omega 2} \kappa^{2} / \sqrt{\beta^{*}}
\end{gathered}
$$

With the following definitions:

$$
\nu_{t}=\frac{k}{\omega}
$$




$$
\begin{gathered}
\tau_{i j}=\mu_{t}\left(\frac{\partial u_{i}}{\partial x_{j}}+\frac{\partial u_{j}}{\partial x_{i}}-\frac{2}{3} \frac{\partial u_{k}}{\partial x_{k}} \delta_{i j}\right)-\frac{2}{3} \rho k \delta_{i j} \\
F_{1}=\tanh \left(\arg _{1}^{4}\right) \\
\arg _{1}=\min \left[\max \left(\frac{\sqrt{k}}{0.09 \omega y} ; \frac{500 \nu}{y^{2} \omega}\right) ; \frac{4 \rho \sigma_{\omega 2} k}{C D_{k \omega} y^{2}}\right]
\end{gathered}
$$

where $y$ is the distance to the next surface and $C_{k \omega}$ is the positive portion of the cross-diffusion term of Eq. (A2):

$$
\mathrm{CD}_{k \omega}=\max \left(2 \rho \sigma_{\omega 2} \frac{1}{\omega} \frac{\partial k}{\partial x_{j}} \frac{\partial \omega}{\partial x_{j}}, 10^{-20}\right)
$$

The term $\arg g_{1}$ obviously goes to zero far enough away from solid surfaces because of the $1 / y$ or $1 / y^{2}$ dependency in all three terms in Eq. (A9). Inside a boundary layer the three arguments in Eq. (A9) have the following purpose: the first argument is the turbulent length scale divided by $y$. It is equal to 2.5 in the log layer and goes to zero towards the boundarylayer edge. The second argument ensures that $F_{1}$ is equal to one in the sublayer [note that $\omega$ goes like $1 / y^{2}$ in the near wall region and is proportional to $1 / y$ in the log region, so that $1 /\left(\omega y^{2}\right)$ is constant near the surface and goes to zero in the log region]. The third argument is an additional safeguard against the freestream-dependent solution. It can be shown that the

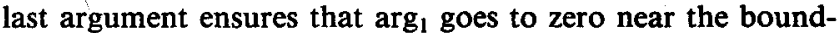
ary-layer edge in case the "degenerate" solution given in Ref. 6 is approached. As $\arg _{1}$ goes to zero near the boundary-layer edge, so does $F_{1}$ so that the standard $k-\epsilon$ is used in that region.

The following choice of freestream values is recommended:

$$
\omega_{\infty}=(1 \rightarrow 10) \frac{U_{\infty}}{L}, \quad \nu_{t \infty}=10^{-(2-5)} \nu_{\infty}, \quad k_{\infty}=\nu_{t \infty} \omega_{\infty}
$$

where $L$ is the approximate length of the computational domain.

The boundary condition for $\omega$ at a solid surface is:

$$
\omega=10 \frac{6 \nu}{\beta_{1}\left(\Delta y_{1}\right)^{2}} \text { at } y=0
$$

where $\Delta y_{1}$ is the distance to the next point away from the wall. Equation (A12) simulates the smooth wall boundary condition of Ref. 1 as long as $\Delta y_{1}^{+}<3$.

\section{Shear-Stress Transport Model}

The SST model is identical to the preceding formulation, except that the constants, $\phi_{1}$, have to be changed to:

Set 1 (SST inner):

$$
\begin{aligned}
& \sigma_{k 1}=0.85, \quad \sigma_{\omega 1}=0.5, \quad \beta_{1}=0.0750, \quad a_{1}=0.31 \\
& \beta^{*}=0.09, \quad \kappa=0.41, \quad \gamma_{1}=\beta_{1} / \beta^{*}-\sigma_{\omega 1} \kappa^{2} / \sqrt{\beta^{*}}
\end{aligned}
$$

and the eddy viscosity is defined as:

$$
\nu_{t}=\frac{a_{1} k}{\max \left(a_{1} \omega ; \Omega F_{2}\right)}
$$

where $\Omega$ is the absolute value of the vorticity. $F_{2}$ is given by:

$$
F_{2}=\tanh \left(\arg _{2}^{2}\right)
$$

$$
\arg _{2}=\max \left(2 \frac{\sqrt{k}}{0.09 \omega y} ; \frac{500 \nu}{y^{2} \omega}\right)
$$

Important detail!

In applying this model, it is important that the reader be aware of the following ambiguity in the formulation of the production term of $\omega$ for the SST model. The definition of the production term of $\omega$ is sometimes written as:

$$
P_{\omega}=\gamma \frac{\omega}{k} \tau_{i j} \frac{\partial u_{i}}{\partial x_{j}}
$$

which introduces the nondimensional group $\nu_{t}(\omega / k)$ in front of the strain rate tensor. In the original and in the BSL model this group is equal to one and the two formulations for $\boldsymbol{P}_{\omega}$ are therefore identical. This is not the case for the SST model because of Eq. (A14). The SST model has been calibrated with respect to Eq. (A2) and Eq. (A17) should therefore not be used.

\section{Acknowledgments}

The author wants to thank S. E. Rogers and J. Bardina for providing the mean flow solvers for the incompressible and the transonic computations, respectively, as well as their assistance in implementing the turbulence models.

\section{References}

'Wilcox, D. C., "Reassessment of the Scale-Determining Equation for Advanced Turbulence Models," AIAA Journal, Vol. 26, No. 11 1988, pp. 1299-1310.

${ }^{2}$ Michelassi, V., and Shih, T.-H., "Elliptic Flow Computation by Low Reynolds Number Two-Equation Turbulence Models," NASA TM-105376, CMOTT-91-11, Dec. 1991.

${ }^{3}$ Wilcox, D. C., "Comparison of Two-Equation Turbulence Models for Boundary Layers with Pressure Gradient," AIAA Journal, Vol. 31, No. 8, 1993, pp. 1414-1421.

${ }^{4}$ Wilcox, D. C., "A Half Century Historical Review of the $k-\omega$ Model," AIAA Paper 91-0615, Jan. 1991.

${ }^{5}$ Huang, P. G., Bradshaw, P., and Coakley, T. J., "Assessment of Closure Coefficients for Compressible-Flow Turbulence Models," NASA TM-103882, March 1992.

${ }^{6}$ Menter, F. R., "Influence of Freestream Values on $k-\omega$ Turbulence Model Predictions," AIAA Journal, Vol. 30, No. 6, 1992, pp. 1651-1659.

${ }^{7}$ Rodi, W., and Scheurer, G., "Scrutinizing the $k-\epsilon$ Model Under Adverse Pressure Gradient Conditions,' Journal of Fluids Engineering, Vol. 108, June 1986, pp. 174-179.

${ }^{8}$ Johnson, D. A., and King, L. S., "Mathematically Simple Turbulence Closure Model for Attached and Separated Turbulent Boundary Layers," AIAA Journal, Vol. 23, No. 11, 1985, pp. 1684-1692.

${ }^{9}$ Menter, F. R., "Zonal Two Equation $k$ - $\omega$ Turbulence Models for Aerodynamic Flows," AIAA Paper 93-2906, July 1993.

${ }^{10}$ Menter, F. R., "Performance of Popular Turbulence Models for Attached and Separated Adverse Pressure Gradient Flows," $A I A A$ Journal, Vol. 30, No. 8, 1992, pp. 2066-2072.

${ }^{11}$ Driver, D. M., "Reynolds Shear Stress Measurements in a Separated Boundary Layer," AIAA Paper 91-1787, June 1991.

${ }^{12}$ Rodi, W., "Experience with a Two-Layer Model Combining the $k-\epsilon$ Model with a One-Equation Model Near the Wall," AIAA Paper 91-0216, Reno, NV, Jan. 1991.

${ }^{13}$ Rogers, S. E., and Kwak, D., “An Upwind Differencing Scheme for the Time-Accurate Incompressible Navier-Stokes Equations," AIAA Paper 88-2583, June 1988.

${ }^{14}$ Rogers, S. E., private communication. NASA Ames, 1993.

${ }^{15}$ Launder, B. E., and Sharma, B. I., "Application of the EnergyDissipation Model of Turbulence to the Calculation of Flow near a Spinning Disk," Letters in Heat and Mass Transfer, Vol. 1, No. 1, 1974, pp. 131-138.

${ }^{16}$ Samuel, A. E., and Joubert, P. N., "A Boundary Layer Developing in an Increasingly Adverse Pressure Gradient," Journal of Fluid Mechanics, Vol. 66, Pt. 3, 1974, pp. 481-505.

${ }^{17}$ Driver, D. M., and Seegmiller, H. L., "Features of a Reattaching Turbulent Shear Layer in Divergent Channel Flow," AIAA Journal, Vol. 23, No. 2, 1985, pp. 163-172.

${ }^{18}$ Thangam, S., and Speciale, C. G., "Turbulent Separated Flow Past a Backward-Facing Step: A Critical Evaluation of Two-Equation Turbulence Models," Institute for Computer Applications in Science and Engineering, ICASE Rept. 91-23, 1991. 
${ }^{19}$ Abid, R., Speciale, C. G., and Thangam, S., "Application of a New $k-\tau$ Model to Near Wall Turbulent Flows," AIAA Paper 910614, Jan. 1991.

${ }^{20}$ Coles, D., and Wadock, A. J., "Flying-Hot-Wire Study of Flow Past an NACA 4412 Airfoil at Maximum Lift," AIAA Journal, Vol. 17, No. 4, 1979, pp. 321-328.

${ }^{21}$ Rogers, S. E., Wiltberg, N. L., and Kwak, D., "Efficient Simulation of Incompressible Viscous Flow Over Single and Multi-Element Airfoils," AIAA Paper 92-0405, Jan. 1992.

${ }^{22}$ Bachalo, W. D., and Johnson, D. A., "An Investigation of Tran- sonic Turbulent Boundary Layer Separation Generated on an Axisymmetric Flow Model,' AIAA Paper 79-1479, June 1979.

${ }^{23}$ Rogers, S. E., Menter, F. R., Mansour, N. N., and Durbin, P. A., "A Comparison of Turbulence Models in Computing Multi-Element Airfoil Flows," AIAA Paper 94-0291, Jan. 1994.

${ }^{24}$ Menter, F. R., "Assessment of Higher Order Turbulence Models for Complex 2D and 3D Flow Fields," Proceedings of the Second International Symposium on Engineering Turbulence Modeling and Measurements (Florence, Italy), Elsevier, Amsterdam, 1993, pp. 229-239.

\title{
Procedure Writing Principles and Practices
}

\author{
Douglas Wieringa, Christopher Moore, and Valerie Barnes
}

Procedures are instructions, and this book explains how to write instructions so that others can understand them. Procedures can range from simple to complex; they describe anything from booting up a personal computer to operating a nuclear power plant during an emergency. Plans, mission statements, proposals, and technical articles are not procedures, although parts of these documents may be considered procedures if they present instructions. No matter how simple or complex the procedure is, certain

1993, 211 pages, Paperback ISBN 0-935470-68-9, \$29.95, Order \#: PPP-1(945)
Place your order today! Call 1-800/682-AlAA

\section{OALAA}

American Institute of Aeronautics and Astronautics

Publications Customer Service, 9 lay Gould Ct., P.O. Box 753, Waldorf, MD 20604 FAX 301/843-0159 Phone 1-800/682-2422 8 a.m. - 5 p.m. Eastern principles govern the way it should be written. The authorsdraw on theirmore than ten years of experienceand present theirprinciples in this book. 